\title{
Die Herren von Heinriet - ihre verschwundene Burg und ihre vergessene Stadt
}

\author{
Von Hermann Ehmer
}

\section{Die Herren von Heinriet und die Staufer}

Die Herren von Heinriet ${ }^{1}$ werden von 1137 bis 1462 urkundlich bezeugt. Die ältesten schriftlichen Belege für die Herren von Heinriet verdanken wir jedoch dem Codex Hirsaugiensis, einer wohl in der zweiten Hälfte des 12. Jahrhunderts entstandenen Handschrift des Klosters Hirsau, die vor allem auch ein Verzeichnis der traditiones, der Schenkungen und Erwerbungen des Klosters enthält. Der Codex liegt bislang nur in einer älteren Ausgabe vor ${ }^{2}$.

In den undatierten Eintragungen des Codex ${ }^{3}$ wird ein Gerungus de Hobenrieth genannt, ferner ein Hugo de Habenrieth et fratres eius Gerung et Rudolff sowie ein Rudiger[o] de Heinrieth. An einer weiteren Stelle des Codex ${ }^{4}$ findet sich noch die Schenkung von zwei Huben in Hohenriet (gemeint ist wohl Unterheinriet) an das Kloster Hirsau durch Irmingart, Ehefrau des Drutwin von Bellheim. Versucht man, die hier genannten Angehörigen der Familie von Heinriet und die Verwandten von Bellheim (Bellenheim) in einen Zusammenhang zu bringen, ergibt sich der folgende Stammbaum:

${ }^{1}$ Einen kurzen Abriss der Geschichte der Herren von Heinriet enthält der Ortsartikel Unterheinriet in: Beschreibung des Oberamts Weinsberg, hg. von dem Königlichen statistisch-topographischen Bureau, Stuttgart 1861, S.361-371, hier S.369-371. Vgl. ferner: Eberhard Schweizer, Ein Gang durch die Geschichte der Herren von Heinriet, in: Untergruppenbach. Heimatbuch der Gemeinde Untergruppenbach, bearb. von Wilfried SeHM, Stuttgart 1992, S. 195-205.

2 Codex Hirsaugiensis (Württembergische Geschichtsquellen, Bd.1), hg. von E[ugen] SchneIder, Stuttgart 1887. Eine Neubearbeitung durch Sönke Lorenz $(\dagger)$ und Stephan Molitor ist angekündigt.

${ }^{3}$ Die nachstehend zitierten Eintragungen finden sich ebd., fol. $49 \mathrm{a}-\mathrm{b}$.

${ }^{4}$ Ebd., fol. 43 a. 


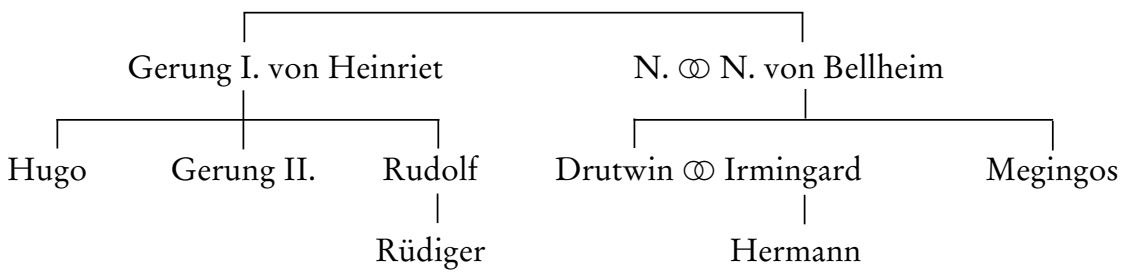

Zur zeitlichen Ansetzung der verschiedenen Besitzübergaben gibt es nur einen einzigen Anhaltspunkt, nämlich die Nennung des Grafen Albrecht von Calw. Gemeint ist damit sehr wahrscheinlich Albrecht IV., der nach seinen Auseinandersetzungen mit Welf VI. wieder im Besitz Calws und der Klostervogtei gekommen war ${ }^{5}$. Somit wären also diese Vorgänge ungefähr auf das Jahr 1140 anzusetzen. Man wird also in dem 1139 urkundlich erscheinenden Helferich von Heinriet ${ }^{6}$, einen Verwandten, womöglich einen Bruder des Heinrieters sehen müssen, den wir hier als Gerung II. bezeichnen. Urkundlich erscheint ein Gerung von Heinriet 1181, der aber einer späteren Generation angehört.

In den älteren urkundlichen Erwähnungen erscheinen die Heinrieter im Gefolge der staufischen Könige und Kaiser. Man hat sie deshalb als staufische Reichsministeriale bezeichnet ${ }^{7}$. Unter Ministerialen werden aber Dienstleute verstanden, die ursprünglich aus unfreiem Stand stammend im Dienste eines Höheren zu wichtigen Stellungen gelangen und damit auch in einen höheren Stand aufsteigen konn$\operatorname{ten}^{8}$. Dies ist nun gerade bei den Heinrietern nicht der Fall. Sie werden in den Zeugenreihen der Urkunden nicht zu den Ministerialen, sondern von Anfang an zu den liberi, den Edelfreien gezählt ${ }^{9}$. Gleichwohl muss gefragt werden, wie die Heinrieter in diese enge Verbindung zu den Staufern gelangen konnten.

${ }^{5}$ Christoph Friedrich STÄLIN, Wirtembergische Geschichte, 2. Teil, Stuttgart/Tübingen 1847, S. $371 \mathrm{f}$.

6 MGH DD K III, 18.

7 Karl Boss, Die Reichsministerialität der Salier und Staufer (MGH, Bd.10, Teil 1-2), Stuttgart 1950-1951, hier Teil 2, S.374.

8 Josef Fleckenstein, Das Rittertum der Stauferzeit, in: Die Zeit der Staufer. Geschichte - Kunst - Kultur [Ausstellungskatalog], Bd.3, Stuttgart 1977, S. 103-109, hier S. 104 f.

${ }^{9}$ Dies ist schon bei der ersten urkundlichen Nennung eines Heinrieters der Fall, nämlich in der Urkunde König Konrads III. von 1139 für das Stift Frankenthal, MGH DD K III, 18. Helfericus von Heinriet (Hehenrith) erscheint hier unter den Edelfreien (liberi) zwischen Walter und Engelhard von Lobenhausen einerseits und Marquard von Grumbach andererseits. - Auf diesen Sachverhalt weist bereits hin: Christoph Jacob Kremer, Abhandlung von den graven von Loewenstein, aeltern und mittlern geschlechts, aus urkunden, in: Historia et Commentationes Academiae Electoralis Scientiarum et Elegantiorum Literarum Theodoro-Palatinae, Vol. I, Mannheim 1766, S.340, Anm. y: „Die von Hehenriet gehoerten zum hohen Adel, und waren dinastae." Ebenso auch STÄLIN (wie Anm. 5) S. 535. 
Die Heinrieter stehen offenbar in Verbindung mit dem Reichsgut Ilsfeld, dessen Zusammenhang mit dem Königtum bis in die fränkische Zeit zurückgeht, wie an verschiedenen Merkmalen abgelesen werden kann. Die „Urkirche“ dieses Königsgutsbezirks war die Michaelskirche auf dem Wunnenstein; in diesen Bezirk gehörten auch die beiden durch die Himmelsrichtungen bezeichneten Orte (Neckar-) Westheim und Auenstein $(=\text { Ostheim })^{10}$. Der Salierkaiser Heinrich IV. schenkte mit einer in Speyer ausgestellten Urkunde vom 15. Februar $1102^{11}$ das Gut Ilsfeld (predium quoddam Ilisfelt) zum Heil seiner Seele und der seiner Vorfahren und seiner Familie der Speyerer Kirche. In diesem Vermächtnis bestimmte der Kaiser, dass am Jahrtag der Kaiserin Gisela, seiner Großmutter, dem Bischof und allen, die an den Gottesdiensten mitwirkten, eine Mahlzeit gereicht und außerdem 200 Arme gespeist werden sollten ${ }^{12}$. Über Ilsfeld erfährt man nur wenig, nämlich dass es im Schozachgau, in der Grafschaft des Adelbert liegt. Es handelt sich eben um das Gut und alles was dazugehört, wobei dieses Zubehör mit der üblichen Pertinenzformel beschrieben wird. Es gehören auch dazu die Leibeigenen (mancipia) beiderlei Geschlechts, ausgenommen wird jedoch der Hof Jendan oder Gendach ${ }^{13}$, der schon an die Kirche in Sinsheim vergeben war.

Wenige Jahrzehnte später ging das Gut Ilsfeld wieder zurück an den König. Der Staufer Konrad III. (1137-1152) bedrängte den Bischof Günther von Speyer, seinem Sohn Heinrich $(† 1150)$ das Gut Ilsfeld als Lehen zu übergeben. Wir haben Kenntnis davon erst durch eine in Speyer ausgestellte Urkunde des Bischofs Günter vom 13. März $1157^{14}$, in der er die Stiftung Heinrichs IV. ausführlich darstellt, ebenso aber auch, dass er dem Drängen König Konrads nicht freiwillig nachgegeben habe. Dieses hatte offensichtlich auch deswegen Erfolg, weil es Konrad III. schon früh gelang, die in Verbindung mit dem Königsgut Ilsfeld stehenden Adligen an sich zu ziehen, wie an Helfrich von Heinriet zu sehen ist.

10 Hermann Ehmer, Der Gleißende Wolf von Wunnenstein. Herkunft, Karriere und Nachleben eines spätmittelalterlichen Adeligen (Forschungen aus Württembergisch Franken, Bd.38), Sigmaringen 1991, S.19-24.

11 WUB 1, Nr. 262, S. 331 f. Vgl. die genannten Belege hier und im Folgenden auch in der Online-Ausgabe unter www.wubonline.de (Abruf: 18.12.2018).

12 Eine entsprechende Eintragung findet sich in der Speyerer Totenbuchüberlieferung zum 15. Februar: „Gisela imperatrix obiit, pro cuius anime remedio Heinricus III. imperator constituit dari de Jlesvelt servitium fratribus omnibus de claustris et ducentos pauperes pasci;“ Hansjörg Grafen, Forschungen zur älteren Speyerer Totenbuchüberlieferung. Mit einer Textwiedergabe der Necrologanlage von 1273 (Quellen und Abhandlungen zur mittelrheinischen Kirchengeschichte, Bd.17), Mainz 1996, S.287. - Gisela starb 1046.

${ }^{13}$ Der abgegangene Ort Gendach lebt bis zum heutigen Tag noch als Flurname auf der Gemarkung Ilsfeld weiter.

14 WUB 2, Nr. 357, S. 106-108. 
Die Verbindung der Heinrieter mit den Staufern vererbte sich auf die folgenden Generationen, denn im Gefolge Kaiser Friedrichs I. erscheint $1181^{15}$ und $1186^{16}$ Gerung von Heinriet. Man wird annehmen müssen, dass es derselbe Gerung ist, den wir hier Gerung III. nennen, der noch 1190 in Kaiserslautern ${ }^{17}$ und 1192 in Worms ${ }^{18}$ für Kaiser Heinrich VI. als Zeuge erscheint. Ein Konrad von Heinriet ist 1222 Zeuge, als Heinrich von Langenburg in Wimpfen in Gegenwart von König Heinrich (VII.) den Verkauf seiner ihm durch seine Frau zugebrachten Güter in Bieringen an das Kloster Schöntal beurkundet ${ }^{19}$. Dieser Konrad erscheint in der Zeugenreihe nach Hartmann und Ludwig von Württemberg, dem Notar Marquard und Konrad und Ulrich von Dürn an sechster Stelle. Es ist wohl derselbe Konrad, den wir hier Konrad I. nennen, der bereits in einer Urkunde des Wormser Domkapitels von 1182 genannt wird ${ }^{20}$. Mit König Heinrich (VII.), dem Sohn Kaiser Friedrichs II., nimmt die Königsnähe der Heinrieter ein Ende. Eine die Jahrhunderte überdauernde Erinnerung an die Königsnähe sind zweifellos die staufischen Königsnamen Konrad, Heinrich und Friedrich, die bis an ihr Ende als die häufigsten Vornamen der Heinrieter aufscheinen.

Nach dem Ende der Staufer war die Familie von Heinriet an den territorialen Auseinandersetzungen in Schwaben und Franken beteiligt und darauf angewiesen, sich nach Möglichkeit an Mächtigere anzulehnen. Darauf deutet die Tatsache, dass Konrad II. von Heinriet 1253 als Zeuge für Markgraf Rudolf von Baden erscheint, als dieser gegenüber dem Abt von Salem eine Verzichtserklärung abgibt, wobei es um Güter des Klosters geht, auf die der Markgraf von seinen Vorfahren her Besitzansprüche geltend machen konnte ${ }^{21}$. Die Urkunde wurde auf der Burg Reichenberg im Murrtal ausgestellt, damals Besitz der Markgrafen, ebenso wie Besigheim, dessen Vogt Rugger ebenfalls als Zeuge erscheint. Konrad von Heinriet wird als erster Laie in der Reihe von insgesamt elf Zeugen genannt, unter denen auch Emhard $^{22}$ von Ilsfeld erscheint, der wohl auch als ursprünglich königlicher Gefolgsmann anzusehen ist.

Die Heinrieter erscheinen auch im Umkreis der Herren von Weinsberg, so 1279 als Konrad d. Ä. und Konrad d.J. von Weinsberg Abt und Konvent von Schöntal (Schontal) ihren Hof in Binswangen verkaufen ${ }^{23}$. Auch hier steht Konrad von Heinriet in der Zeugenreihe als erster unter den nobiles, dem Albrecht von Ebers-

\footnotetext{
15 MGH DDF I, 810.

16 MGH DDF I, 954.

17 Regesta Imperii IV,3 n. 106.

18 Regesta Imperii IV, 3,1 n. 244.

19 StAL B 503 I U 247.

20 WUB 2, Nr. 431, S.220f.

21 Regesten der Markgrafen von Baden und Hachberg, Bd.1, Nr.419, S. 36.

22 Die Lesung Einhard in den Regesten der Markgrafen von Baden und Hachberg (wie Anm. 21) ist zu verbessern.

23 StAL B 503 I U 297.
} 
berg, Erkinger von Magenheim, Vater und Sohn, und Engelhard von Weinsberg folgen. Bei Konrad d. Ä. muss es sich um Konrad II. handeln, während der Jüngere, offensichtlich sein Sohn, als Konrad III. bezeichnet werden muss. Dieser Konrad dictus de Hebenriet bestätigte 1277 den Verkauf von Äckern oder anderen Gütern in Gattenhofen durch seine Eigenleute Heinrich Guderius und Geizebart von Gattenhofen an das Dominikanerinnenkloster in Rothenburg ${ }^{24}$. Wir sehen hier einen Heinrieter erstmals fernab des Ursprungs der Familie begütert ${ }^{25}$. Dieser Besitz ist wohl durch Heirat an Konrad III. gelangt.

\section{Die verschwundene Burg Heinriet}

Über die Stelle, an der die Burg Heinriet einst stand, herrscht einigermaßen Unklarheit. Man möchte sie eigentlich, wie dies schon die Oberamtsbeschreibung Weinsberg von $1861^{26}$ tut, auf dem Berg über dem Dorf Unterheinriet vermuten, das zwischen dem Oberlauf der Schozach und dem hier einmündenden Buchbach liegt. Die Vermutung erscheint sinnvoll, in Unterheinriet den am Fuße des mutmaßlichen Burgbergs entstandenen Burgweiler zu anzunehmen. Der Ort wurde ja zunächst nach der Burg Heinriet ${ }^{27}$ genannt, hieß aber alsbald Unterheinriet, zur Unterscheidung von der weiter oberhalb an der Schozach angelegten Ausbausiedlung, die den Namen Oberheinriet erhielt ${ }^{28}$.

Die Erhebung über dem Dorf Unterheinriet führt heute den Namen Höhberg, ein typischer Weinbergsname. In der Tat sind die nach Süden und Westen weisenden Hänge des Berges mit Reben bestockt. Dies würde dann auch erklären, weshalb von der Burg keine Überreste mehr zu sehen sind, da ihre Steine alsbald nach ihrer Zerstörung anderwärts, nicht zuletzt in den Terrassenmauern der Weinberge verbaut werden konnten. Freilich gibt es auf diesem Berg auch eine heute noch zu sehende Steingrube, die vermutlich alt ist. Aus dieser könnten nicht nur Steine für

${ }^{24}$ Die Urkunden der Reichsstadt Rothenburg 1182-1400 (Veröffentlichungen der Gesellschaft für Fränkische Geschichte Bd.III, 6), bearb. von Ludwig Schnurrer, Neustadt/ Aisch 1999, Nr. 84, S. 42.

${ }_{25}$ Gattenhofen im Landkreis Ansbach.

26 OAB Weinsberg, S.364. - So noch der Verfasser in: Der Landkreis Heilbronn, Bd.2, hg. vom Landesarchiv Baden-Württemberg in Verbindung mit dem Landkreis Heilbronn, Stuttgart 2010, S. 443. - Das Land Baden-Württemberg, Bd.4: Regierungsbezirk Stuttgart, Regionalverbände Franken und Ostwürttemberg, hg. von der Staatlichen Archivverwaltung Baden-Württemberg, Stuttgart 1980, S. 99 enthält sich einer Lokalisierung der einstigen Burg Heinriet.

27 So in der Urkunde des Papstes Alexander IV. von 1257 für das Kloster Lichtenstern, wo von der villa que Hehenriet vulgariter nuncupatur die Rede ist; WUB 5, Nr.1310, S. 75-77 mit Bd.11, S. 576.

28 Der Name Oberheinriet (in superiori Habenriet) findet sich bereits 1247 in der Urkunde des Papstes Innozenz IV. für das Stift Oberstenfeld; WUB 4, Nr. 1098, S. 160. 
die Weinbergsmauern, sondern auch die Steine für einen Burgbau gewonnen worden sein. Allerdings weist der sanft abfallende Berg nirgends eine Stelle auf, an dem eine Burg in Spornlage oder als Abschnittsburg hätte errichtet werden können.

Immerhin könnte der Höhberg einen Hinweis auf die Herkunft des Namens Heinriet geben. Dieser wurde aus einem ursprünglichen Hohen-, Haohen-, Hähen-, Hehenried oder Henheriet zu Heinriet abgeschliffen, wobei die zuletzt genannte Form Henheriet (in verschiedenen Schreibweisen) der heute noch gebräuchlichen mundartlichen Form am nächsten kommt. Das Grundwort -riet oder -ried kann jedoch nicht das mittelhochdeutsche Wort für Schilfrohr oder Riedgras sein, vielmehr geht es hier um einen ,ausgereuteten Grund“29, wobei es offenbleiben muss, ob die Rodung an dieser Stelle nun für einen Weinberg oder den Bau einer Burg erfolgte. Es handelt sich aber hier um einen aus einer Stellenbezeichnung abgeleiteten Burgnamen, anders als die der benachbarten Burgen Stettenfels, Wildeck und Helfenberg, die jüngeren Datums sind. Während bei diesen Namen das Bestimmungwort ein abstrakter Begriff ist, wird dieses bei Heinriet von einem Personennamen Haho abgeleitet ${ }^{30}$.

Die Suche nach der verschwunden Burg Heinriet bleibt auf dem Höhberg über Unterheinriet also ohne Ergebnis. Überhaupt war der Ort der Burg der Herren von Heinriet schon im 19. Jahrhundert unklar. Nach der bereits erwähnten Oberamtsbeschreibung ${ }^{31}$ verweist „die Volkssage ... sie auf die gegenüberliegende sogen. Kohlebene“, den Höhenrücken zwischen Buchbach und Farnersberger Bach. Es versteht sich, dass diese Annahme von der Oberamtsbeschreibung als irrig abgetan wurde.

Um bei der Lösung dieses Problems weiter zu kommen, sollten die einschlägigen schriftlichen Quellen herangezogen werden, zunächst die Lagerbücher, die im Herzogtum Württemberg in der Zeit der österreichischen Verwaltung in den 1520er Jahren angelegt worden sind und für viele Gebiete des Landes eine flächendeckende Erfassung der Flur- und Stellennamen bieten. Da Unterheinriet damals zum Amt Beilstein gehörte, müsste also das betreffende Lagerbuch, das aus dem Jahr 1524 stammt, Aufschluss in der Frage nach der verschwundenen Burg Heinriet geben können ${ }^{32}$. Es findet sich dort unter Unterheinriet aber nur der bereits bekannte Hörberg, vermutlich identisch mit dem dort ebenfalls erwähnten $\mathrm{Heg}$ berg $^{33}$. Ein Hinweis auf die Burg Heinriet findet sich hier jedoch nicht.

Es muss nun aber auch ein anderer Ort in die Suche nach der Burg Heinriet einbezogen werden, nämlich der knapp $3 \mathrm{~km}$ oberhalb von Unterheinriet über dem

${ }^{29}$ Vgl. Lutz Reichardt, Ortsnamenbuch des Ostalbkreises, Teil II: M-Z (VKgL B 140) Stuttgart 1999, S. 311 zu Wustenriet.

30 Das Land Baden-Württemberg (wie Anm.26), S. 98.

31 OAB Weinsberg, S. 364.

${ }^{32}$ HStAS H 101/5 Bd. 1, hier zitiert nach der Edition: Altwürttembergische Lagerbücher aus der österreichischen Zeit VI, bearb. von Thomas Schulz (VKgL A 28) Stuttgart 1991.

33 Altwürttembergische Lagerbücher (wie Anm. 32) S. 97. 
rechten Hang des Buchbachs gelegene Weiler Vorhof. Der Name deutet doch wohl darauf hin, dass es sich hier um den Wirtschaftshof der Burg Heinriet gehandelt hat. Somit dürfte die dazugehörige Burg nicht weit davon entfernt gewesen sein. Schon in der Oberamtsbeschreibung ${ }^{34}$ heißt es: „An der östlichen Seite des Weilers trifft man noch eine mit einem verschütteten Graben umgebene kleine Anhöhe mit Mauerresten eines abgegangenen Burgstadels (suburbium in der Urk. von 1330 ...).“ Das Königreich Württemberg von 1904 sagt dann unmissverständlich: „Reste der Burg [Heinriet] mit Kapelle zur h. Katharina 1330. Hier saß $1139 \mathrm{ff}$. das edelfreie Geschlecht von Heinriet, dessen letzter 1462 fiel, stammverwandt mit den Hacken von Wöllstein, O.A. Aalen, und von Hoheneck (Wappen: 3 runde Scheiben). “ ${ }^{35}$ Allerdings findet sich die Angabe „Reste der Burg und einer Kapelle zur h. Katharina von 1330." auch unter Oberheinriet ${ }^{36}$; ein offenkundiger Druckfehler, der dann gelegentlich für Verwirrung gesorgt hat.

In der Tat ist im Vorhof auch heute noch ein namhafter Mauerrest in Schalenbauweise zu sehen, ferner ein offenbar terrassierter Bergkegel, auf dem immerhin eine Turmburg, ähnlich den benachbarten Burgen Wildeck und Helfenberg, Platz gehabt hätte. Werner Heim hat anhand der Urkatasterkarte von 1834 einen Grundriß der Burg und der westlich davon angelegten Burgsiedlung, des Vorhofs, entworfen ${ }^{37}$ (Abb. 1, 2). Karl-Heinz Dähn hat dann durch Begehung des Geländes die Rekonstruktion Heims erhärtet ${ }^{38}$. Beide - Heim und Dähn - vermuten also die Burg Heinriet beim Vorhof, doch fehlt ein letzter Beweis dafür (Abb.3). Denn merkwürdig ist trotz der genannten Befunde und der Tatsache, dass heutige Bewohner des Vorhofs noch von einem Burggraben reden und es im Ort einen Burgplatz gibt, dass der Name Heinriet hier nicht haften blieb, sondern lediglich die Bezeichnung Vorhof.

Den fehlenden Beleg vermag nun die bisher unterbliebene Heranziehung des Lagerbuchs von 1524 mit den dort genannten Flur- oder Stellennamen des Vorhofs zu erbringen. Es wird hier die Hofstatt genannt, die offenbar auf den Wirtschaftshof hindeutet. Es wird hier ferner ein Burggraben erwähnt, ebenso ein alter Burgstall. Es findet sich dann noch die Bezeichnung hinder dem alten Schloß und schließlich als endgültiger Beweis: genannt Heberiet, alter Burgstall ${ }^{39}$. Damit ist hinlänglich bewiesen, dass die verschwundene Burg Heinriet einst beim Vorhof stand.

34 OAB Weinsberg, S.368 f.

35 Das Königreich Württemberg. Eine Beschreibung nach Kreisen, Oberämtern und Gemeinden, hg. von dem Kgl. Statistischen Landesamt, Bd. 1, Stuttgart 1904, S. 660.

36 Ebd., S.659.

37 Werner Heim, Burg und Vorhof. Zur Lage des Heilbronner Königshofes, in: Schwaben und Franken. Heimatgeschichtliche Beilage der „Heilbronner Stimme“ 7 (1963) Nr. 2.

38 Karl-Heinz DëHN, Unbekanntes Burgenland Löwensteiner Berge, in: Jahrbuch für schwäbisch-fränkische Geschichte 29 (1979/81) S. 95-116. Zum Vorhof vgl. S. 108 f.

39 Altwürttembergische Lagerbücher (wie Anm.32) S. 100 f. 


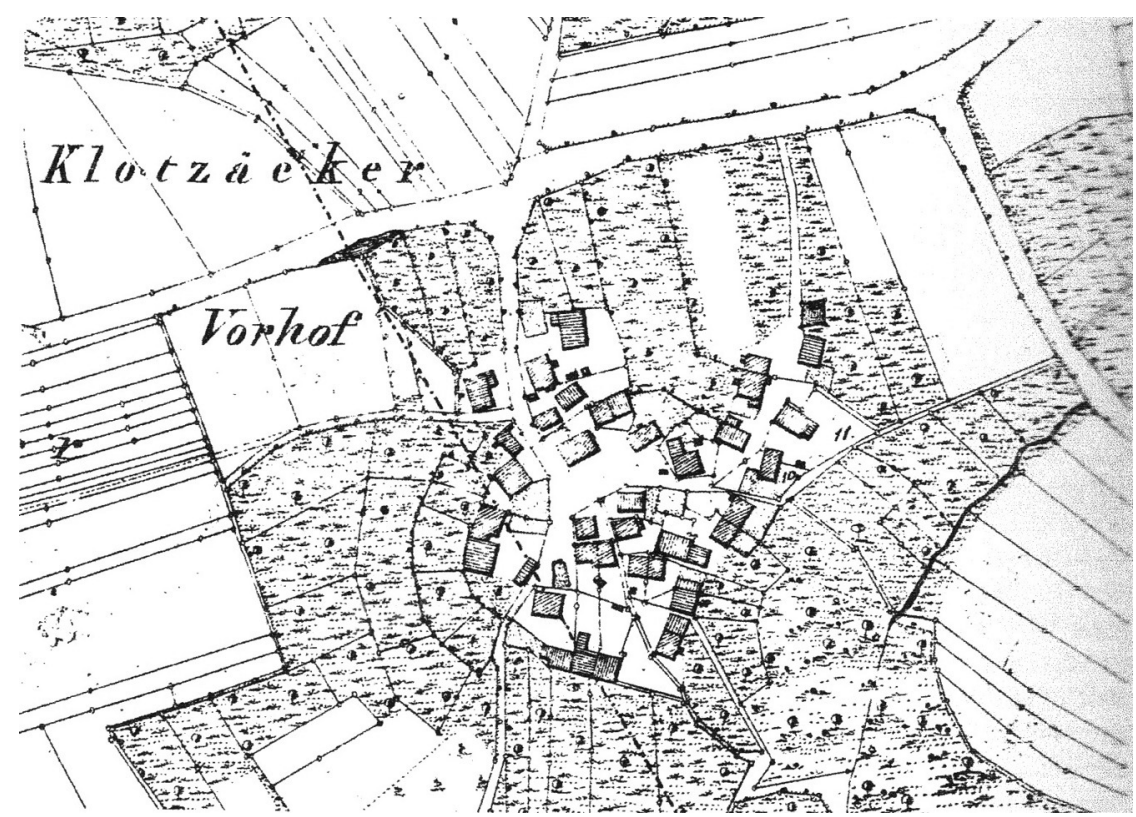

Abb. 1: Ortslage Vorhof (Gem. Unterheinriet) nach der Urkatasterkarte von 1834.

Wenn von der Burg Heinriet im Jahre 1524 nur noch ein Burgstall - bestenfalls also eine Ruine - zeugte, muss gefragt werden, warum die Burg Heinriet im Jahre 1528 vom Schwäbischen Bund als „Schlupfwinkel des Hans Thomas v. Absberg und seiner Genossen“ zerstört wurde, wie die Landesbeschreibung ${ }^{40}$ angibt, die dabei dem Vorgängerwerk, dem Königreich Württemberg ${ }^{41}$ folgt. Geht man der Angabe des Königreichs Württemberg nach und versucht, die Quelle dafür ausfindig zu machen, so stößt man auf die zeitgenössische Dokumentation des Feldzugs des Schwäbischen Bundes von 1523, bei der eine Anzahl von Burgen in Franken zerstört wurde ${ }^{42}$. Heinriet ist dabei aber nicht genannt, weil es ja für den genannten Feldzug völlig abseits $\mathrm{lag}^{43}$.

40 Das Land Baden-Württemberg (wie Anm.26) S. 99.

${ }^{41}$ Das Königreich Württemberg. Eine Bescheibung von Land, Volk und Staat, hg. von dem Kgl. Statistischen Landesamt, Bd.3, Stuttgart 1886, S.254. Ebenso in der folgenden [dritten] Ausgabe des Werkes in Bd.1, S.660.

${ }^{42}$ Vgl. dazu Thomas Steinmetz, Conterfei etlicher Kriegshandlungen von 1523 bis in das 1527 Jar. Zu Burgendarstellungen über die „Absberger Fehde“ oder den „Fränkischen Krieg“, in: Beiträge zur Erforschung des Odenwaldes und seiner Randlandschaften, Bd.4, Breuberg-Neustadt 1986, S.365-386.

${ }^{43}$ Hinweise auf die Burg Heinriet finden sich daher auch in der neueren einschlägigen Literatur nicht, vgl. Peter Ritzmann, „Plackerey in teutschen Landen“. Untersuchungen 
Da das Königreich Württemberg von 1886 ausdrücklich das Jahr 1528 als den Zeitpunkt der Zerstörung der Burg Heinriet angibt, wird dies auf eine mißdeutete Quellenangabe zurückgehen. Als vor 1886 erschienene Quellenveröffentlichungen kommen hier die 1853 von Karl Klüpfel herausgegebenen Urkunden zur Geschichte des Schwäbischen Bun$\operatorname{des}^{44}$ und die 1873 von Joseph Baader edierten Verhandlungen über Thomas von Absberg ${ }^{45}$ in Betracht. Während das Werk von Klüpfel überhaupt keine Hinweise auf Heinriet enthält, nennt Baaders Edition ein Hauriet ${ }^{46}$, das durch seine Lage im Weinsberger Tal eindeutig als unser Heinriet zu identifizieren ist. Der Name wird 1528 in einem Katalog von Fragen genannt, der vier Gefangenen des

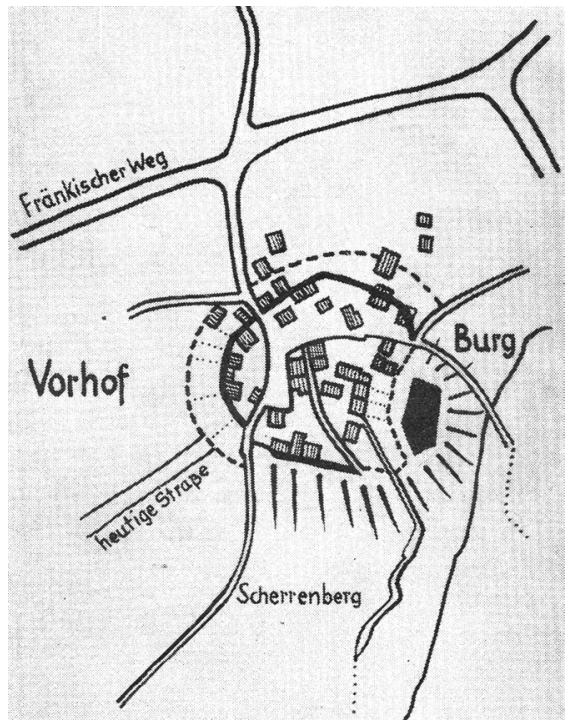

Abb. 2: Lage der Burg Heinriet beim Vorhof (nach Werner Heim, 1963). Bundes im Verhör vorgelegt wurde.

Die betreffende Frage lautete: „Item wie offt sy im weinsperger Thal zu Hauriet bey einem knecht, Wilhelm genannt, so des Jorgleins, Hanns Thomas Knecht pruder ist, gewest seien. “47 Jeder der Verhörten antwortete, „im Weinßperger thal sey er zu Hauriet nit gewest. “48 Die Verbindung Heinriets zu dem Absberger ist also etwas weit hergeholt, sie scheint im weiteren Verlauf der Verhöre auch keine Rolle mehr gespielt zu haben. Gleichwohl hat ein Be- oder Zuarbeiter des Königreichs Württemberg aus dieser Nennung Heinriets zum Jahre 1528 die Zerstörung der

zur Fehdetätigkeit des fränkischen Adels im frühen 16. Jahrhundert und die Reichsstadt Nürnberg, insbesondere am Beispiel des Hans Thomas von Absberg und seiner Auseinandersetzung mit den Grafen von Oettingen (1520-31), München 1995; Horst CarL, Der Schwäbische Bund 1488-1534. Landfrieden und Genossenschaft im Übergang vom Spätmittelalter zur Reformation (Schriften zur südwestdeutschen Landeskunde, Bd.24), Leinfelden-Echterdingen 2000.

${ }^{44}$ Urkunden zur Geschichte des Schwäbischen Bundes, hg. von K. KLÜPfEL, Bd.2, 1507-1533 (Bibliothek des Literarischen Vereins, Bd.31), Stuttgart 1853.

45 Verhandlungen über Thomas von Absberg und seine Fehden gegen den Schwäbischen Bund 1519 bis 1530, hg. von Joseph BAADER (Bibliothek des Literarischen Vereins, Bd. 114), Tübingen 1873.

46 Ebd., S. 451.

47 Ebd., S. 440.

48 Ebd., S. 451, ähnlich S. 443. 
Burg Heinriet herausgelesen. Da dies ein offenkundiger Fehlschluss war, ist es jetzt an der Zeit, die schon über ein Jahrhundert durch die Literatur geschleppte Anga$\mathrm{be}^{49}$ endgültig zu streichen.

Als Ergebnis der Suche nach der verschwundenen Burg Heinriet kann aber hier festgestellt werden, dass diese, wie das Lagerbuch von 1524 mit aller wünschenswerten Deutlichkeit ausweist, beim Vorhof stand. Sie ist schon frühzeitig - jedenfalls vor 1524 - zerstört worden, so dass der Name Heinriet an dieser Stelle nicht mehr haften blieb, sondern lediglich der Vorhof, der womöglich mit der Burg zerstört, aber wieder aufgebaut worden ist. Der Grund, weshalb der Name Heinriet hier in Vergessenheit geriet, ist sicher darin zu suchen, dass dieser von dem heutigen Unterheinriet in Anspruch genommen wurde. Umgekehrt wird man aber davon ausgehen müssen, dass die Herren von Heinriet zunächst in (Unter-)Heinriet ansässig waren und sich danach nannten. Zu einem späteren Zeitpunkt errichteten sie die Burg unmittelbar an der Kante des hier einigermaßen steil zum Buchbach abfallenden Hangs, an der Stelle, wo eine natürliche Klinge einen weiteren Schutz bot. Diese Klinge wurde im 19. Jahrhundert durch den Damm der nach Löwenstein führenden Straße überbrückt, ist aber noch zu beiden Seiten der Straße zu erkennen. Dieser Straßendamm scheint im westlichen Teil des Ortes, bei dem heute so genannten Burgplatz mit Steinen aufgeführt worden zu sein, die von der Burg stammen könnten. Doch zeigen sie keine Bearbeitungsspuren, die mit Sicherheit ins Mittelalter verweisen würden.

\section{Die Heinrieter und die geistlichen Stiftungen in ihrem Umfeld}

Ein Blick auf die Verbindungen der Heinrieter mit den geistlichen Stiftungen in ihrem Umfeld, nämlich das Stift Oberstenfeld, das Dominikanerinnenkloster Steinheim und das Zisterzienserinnenkloster Lichtenstern, vornehmlich in der Stauferzeit, vermag die Stellung der Familie näher zu beleuchten.

Das Stift Oberstenfeld ist die Gründung eines vornehmen Familienverbands des salischen Reichs. Dem Gründungsdatum 1016 kommt eine hohe Wahrscheinlichkeit $\mathrm{zu}^{50}$. Der wohl wichtigste Angehörige der Gründerfamilie des Stifts Oberstenfeld war ein gewisser Udalrich, der als Kanzler der Könige Heinrich II. und Konrad II. wirkte, am 10. September 1032 starb und in der Oberstenfelder Stiftskirche begraben ist.

49 Auch der quellenmäßig fundierte Aufsatz von Schweizer, Ein Gang (wie Anm. 1) hier S. 192, geht von der Zerstörung der Burg 1528 aus. So auch noch der Verfasser in: Der Landkreis Heilbronn (wie Anm. 26) S. 443.

$50 \mathrm{Vgl}$. Ehmer, Das Stift Oberstenfeld von der Gründung bis zur Gegenwart, Tübingen 1998, hier S.60-63. 


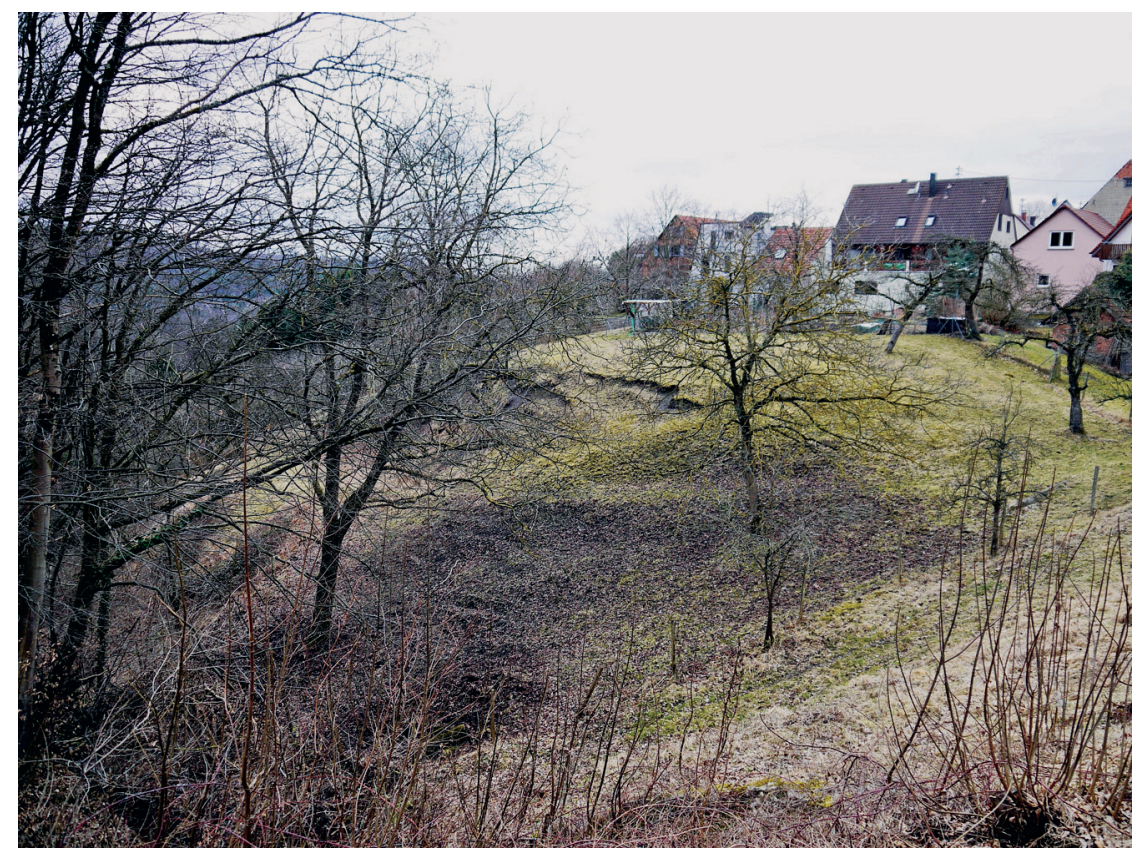

Abb.3: Burghügel beim Vorhof, Ansicht von Nordosten (Aufnahme: Ehmer). 


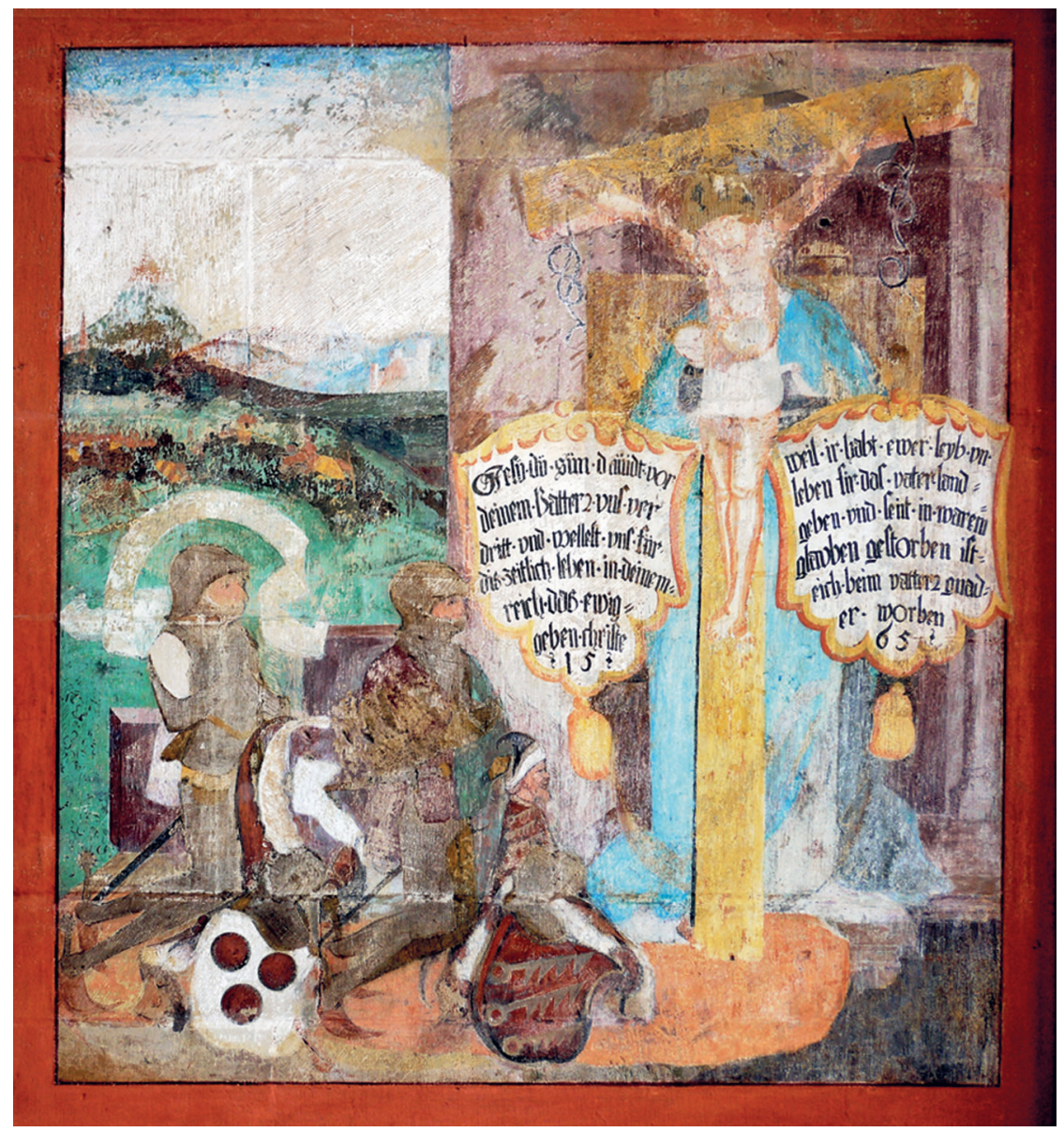

Abb.4: Gedächtnisbild in der Alexanderkirche in Marbach für die am

30. April 1460 in der Pfälzer Fehde gefallenen Konrad von Heinriet (links) und Kaspar Spät (Aufnahme: Ehmer). 
Die Schutzvogtei über das Stift übte anfangs zweifellos die Familie der Gründer aus. Die Nachfolge der Gründerfamilie traten offenbar die Herren von Heinriet an. Der Oberstenfelder Nekrolog nennt nämlich zum 14. April einen Vogt Gerung, zum 19. Mai Agnes die Vögtin ${ }^{51}$. Es liegt nahe, die beiden zusammen zu nehmen und den Vogt mit Gerung III. von Heinriet zu identifizieren, der von 1181-1192 urkundlich genannt wird.

Als Nachfolger der Heinrieter als Vögte des Stifts Oberstenfeld werden im 13. Jahrhundert die Herren von Lichtenberg genannt. Diese standen bis zu ihrem Ende in enger Verbindung mit dem Stift, nicht nur durch die Vogtei, die 1357 an Württemberg überging, vielmehr stellten sie auch Chorfrauen und Äbtissinnen des Stifts $^{52}$. Die Herren von Lichtenberg führten dasselbe Wappen wie die von Heinriet. Da die Heinrieter ebenso wie die Lichtenberger zu den Edelfreien zählten, waren sie auch eines Stammes. Wenn also vermutet werden muss, dass die Lichtenberger eine nicht näher zu bestimmende genealogische Verbindung zu der Grafenfamilie hatten, die das Stift Oberstenfeld gründete, so gilt das in gleicher Weise auch für die Heinrieter. Der Übergang der Schirmvogtei über das Stift Oberstenfeld von den Heinrietern zu den Lichtenbergern dürfte also auf der Verwandtschaft der beiden Familien beruhen.

Es versteht sich, dass bei der engen Verbindung der Heinrieter zum Stift Oberstenfeld auch Frauen aus diesem Hause dem Konvent angehörten. Bei der namentliche Erwähnung von 22 sanctimoniales im Kloster Oberstenfeld zum 11. März 1385 erscheint auch Klara von Heinriet ${ }^{53}$. Diese starb an einem 4. April und hatte zusammen mit ihrer Schwester von Steckelberg dem Stift 3 Morgen Wiesen an der Gänsstiegel für ihre Jahrzeit gestiftet ${ }^{54}$. Die im Oberstenfelder Nekrolog zum 10. Mai eingetragene Jahrzeitstiftung der Chorfrau Adelheid von Sachsenheim, die wohl in die zweite Hälfte des 15. Jahrhunderts zu datieren ist, bestand aus einer Hellergült aus dem Haus der Adelheid zwischen der von Henheriet und der von Dalhen $^{55}$. Es geht daraus hervor, dass die Chorfrauen im Stiftsbezirk eigene Pfründhäuser besaßen, und vermutlich geht das Haus derer von Heinriet auf die eben genannte Chorfrau Klara zurück.

Die Heinrieter betätigten sich selbstverständlich auch als Wohltäter des Stifts Oberstenfeld. Der Nekrolog nennt zum 5. September einen Konrad von Heinriet, der dem Stift einen Hof zu Bottwar schenkte ${ }^{56}$. Ein weiter Konrad von Heinriet, dessen Todestag der 19. Oktober war, vermachte dem Stift einen Hof zu Ottmars-

51 G[ebhard] Mehring, Das Stift Oberstenfeld, in: WVjH 6 (1897) S. 269 und 273.

${ }^{52}$ Enmer, Das Stift Oberstenfeld (wie Anm. 50) hier S.67.

${ }^{53}$ Gerhard Hess, Beiträge zur älteren Geschichte des Frauenstifts Oberstenfeld, in: ZWLG 9 (1949/50) S. 76, Anm. 233.

${ }^{54}$ Mehring (wie Anm. 51) S. 269.

55 Ebd., S. 272.

56 Ebd., S. 281. 
heim ${ }^{57}$. Die zeitliche Ansetzung dieser Stiftungen ist leider nur annähernd ins 12. oder 13. Jahrhundert möglich, weil diese nicht urkundlich belegt sind, sondern nur im Nekrolog vorkommen.

Die zeitliche Einordnung ist bei anderen, mit den Heinrietern verbundenen Schenkungen an das Stift Oberstenfeld besser möglich. Der Zeit vor 1330 gehört folgender Eintrag im Nekrolog an, wo es zum 22. Januar heißt: her Hug von Fleckenstein, Christina sein husfrowe, die gaben uns $10 \mathrm{suf}$ dem hofe zu Rörach ${ }^{58}$. Die Fleckenstein sind im unteren Elsaß beheimatet; Hug von Fleckenstein aus der älteren (Sulzer) Linie seines Geschlechts war vermutlich verheiratet mit Christina von Meckenheim aus einer in der linksrheinischen Pfalz ansässigen Adelsfamilie ${ }^{59}$. Damit stellt sich die Frage, was dieses Ehepaar zu seiner Stiftung im Stift Oberstenfeld veranlasste. Eine Antwort gibt der Nekrolog, der zum 13. April eine Chorfrau Anna von Fleckenstein ${ }^{60}$ erwähnt, die dem Stift eine Gült von 10 Schilling gab. Zum 9. Mai wird eine Christin von Fleckenstein canonica genannt ${ }^{61}$, die $10 \mathrm{lb} \mathrm{h}$ für ein ewiges Seelgerät stiftete. Beide Chorfrauen dürften Töchter des genannten Ehepaars sein ${ }^{62}$. Immerhin erklärt sich mit diesen Nennungen die im Nekrolog zum 14. Juli vermerkte Schenkung des Rudolf von Heinriet und seiner Frau Brigitte von Fleckenstein sowie deren Söhnen Rudolf und Heinrich, die das Stift Oberstenfeld mit zwei Messbüchern und einem Kelch begabten ${ }^{63}$. Bride oder Brigitte von Fleckenstein wird als Tochter des Hug von Fleckenstein und seiner Ehefrau Christine genannt.

Diese Verbindung der Fleckensteiner zum Stift Oberstenfeld wirft zugleich ein Licht auf einen schriftlosen Grabstein, der in der Turmkrypta der Stiftskirche steht und das sechsmal (von grün und silber) geteilte Wappen der Fleckensteiner zeigt, zugleich mit dem Wappen mit den drei Ballen, wobei die Wappenschilder mit den Spitzen zueinander gekehrt sind. Das letztere Wappen könnte das Lichtenberger, aber auch das Heinrieter sein. Man wird sich für die letztere Möglichkeit entscheiden müssen, so dass es sich hier wohl um den Grabstein für Rudolf von Heinriet und seine Ehefrau handelt. Das Ehepaar erscheint in den 1370er Jahren mit Verkäufen in Dallau bei Mosbach; der Ritter Rudolf von Heinriet und seine Frau Bryde von Fleckenstein mit ihren Söhnen Rudolf, Heinrich und Konrad erschei-

57 Ebd., S. 284

58 Ebd., S.263.

59 Europäische Stammtafeln, NF hg. von Detlev Schwennicke, Bd.7, Marburg 1979, Tf. 25; Peter Müller, Die Herren von Fleckenstein im späten Mittelalter (Geschichtliche Landeskunde, Bd.34), Stuttgart 1990, S. 454 f., Stammtafel S. 701.

${ }^{60}$ Mehring (wie Anm. 51) S. 269.

61 Ebd., S. 272.

${ }^{62}$ Dazu: Wolfgang Martin, Vom Habern - ein Niederadelsgeschlecht im Spiegel der Urkunden und anderer Überlieferungen, in: Aus dem alten Erbach (Aus der Geschichte von Stadt und Grafschaft Erbach, Bd.4), Erbach 1993, S.10-135, hier S. 94.

${ }^{63}$ Mehring (wie Anm. 51) S. 277. 
nen gemeinsam zuletzt am 18. März 137964, Rudolf und sein Sohn Konrad werden noch am 16. Dezember 1379 genannt ${ }^{65}$. Es muss daher angenommen werden, dass Bryde inzwischen verstorben war und Rudolf Anfang 1380 gestorben ist. Für ihren mutmaßlichen Grabstein ergibt sich somit ebenso wie für ihre Schenkung eine verhältnismäßig genaue Datierung.

Ein weiterer Vertreter der Familie von Heinriet ist auch der im Nekrolog zum 8. Juli genannte Walther von Wildegge, der dem Stift $20 \mathrm{lb} \mathrm{h} \mathrm{gab}^{66}$. Es handelt sich hier zweifellos um den 1371-1385 genannten Walter von Heinriet, der 1376-1383 das Amt eines Landrichters zu Rothenburg versah und in seiner Rothenburger Verpflichtungsurkunde vom 2. August 1376 der Reichsstadt seine Burg Wildeck öffnete ${ }^{67}$. Insgesamt bezeugen alle diese Nennungen eine enge Verbindung der Herren von Heinriet mit dem Oberstenfelder Damenstift.

In der Kirche in Steinheim an der Murr steht eine Grabplatte, die sich ursprünglich im Kreuzgang des abgegangenen Klosters Mariental zu Steinheim befand. Die Grabplatte zeigt das Heinrieter und ein zweites, ungedeutetes Wappen. Die Inschrift lautet: $\uparrow$ ANNO DOMINI MCCLXXXXVII $†$ BURCSINT DE HEHENRIET NATA IACET HIC TUMULATA ${ }^{68}$. Mit Burcsint lernen wir die erste in den Quellen bezeugte Frau aus dem Hause Heinriet kennen, zugleich weist sie hin auf die Verbindung der Familie zu dem Kloster Steinheim. Burcsint soll die Tochter der Klosterstifterin Elisabeth, Tochter des Ritters Albrecht von Steinheim sein, die in erster Ehe mit Gerung von Heinriet, hier Gerung IV. genannt, verheiratet war. Das Ehepaar wird in einer Urkunde von $1235^{69}$ genannt, in der es um einen Streit zwischen den Grafen von Sulz und den Steinheimern über das Patronatsrecht der Kirche in Steinheim im Speyerer Bistum geht. Der Streit wurde dadurch beigelegt, dass die Grafen von Sulz der Elisabeth das Patronatsrecht durch den Grafen Gottfried von Löwenstein und Gerung von Heinriet, ihrem Ehemann, als Eigentum übergaben.

${ }^{64}$ Mosbacher Urkundenbuch. Stadt und Stift im Mittelalter (Veröffentlichungen der Kommission für geschichtliche Landeskunde in Baden-Württemberg), bearb. von Konrad Krimm unter Mitarbeit von Hans Schadek, Elztal-Dallau 1986, Nr. 180, S. 121.

65 GLAK 43 Nr. 1386.

66 Mehring (wie Anm. 51) S. 276.

${ }^{67}$ Ludwig Schnurrer (Hg.), Die Urkunden der Reichsstadt Rothenburg 1182-1400, Neustadt an der Aisch 1999, Nr.1635, S.645f.

68 Die Inschriften des Landkreises Ludwigsburg, bearb. von Anneliese Seeliger-Zeiss und Hans Ulrich SchёFеr (Die Deutschen Inschriften, Bd.25), Wiesbaden 1986, Nr.11, S. 12 mit Abb. 12.

69 WUB 3, Nr. 860, S.358f. - In einer Aufzeichnung der Jahrtage des Klosters Oberstenfeld wohl aus der Zeit um 1500 wird der Heinrieter, mit dem Elisabeth von Blankenstein, Tochter des Ritters Albrecht von Steinheim, in zweiter Ehe verheiratet war, Konrad genannt; HStAS H 14 Bd.237, Bl.159. Man wird wohl dem Wortlaut der Urkunde glauben müssen, wonach es sich um Gerung von Heinriet handelte. 
Bei der Urkunde, die in Langenau (bei Ulm) ausgestellt wurde, beeindrucken die verhältnismäßig hochrangigen Zeugen, nämlich Propst Bertold von Aquileja, Propst Konrad zur Hl. Dreifaltigkeit in Speyer, ferner Bertold von Hohenhart, ein weiterer Speyerer Kanoniker. Von den Laien wird nur Graf Otto von Eberstein erwähnt. Man wird daher annehmen können, dass diese Rechtshandlung bereits als Vorbereitung für eine geplante Klostergründung gedacht war ${ }^{70}$. Diese fand freilich erst nach dem Tod Gerungs statt, als sich seine Witwe Elisabeth mit Bertold von Blankenstein, einem Vertrauten des Grafen Ulrich von Württemberg verheiratet hatte. Die Klostergründung sollte nun eine Rolle im Ausbau des Territoriums des Grafen von Württemberg spielen. Dieser reiste 1251 nach Lyon, wobei er die päpstliche Genehmigung für die geplante Klostergründung einholte, die auch umgehend ins Werk gesetzt wurde. Die Steinheimer Pfarrkirche wurde 1255 dem Kloster inkorporiert, 1261/1262 wurde das Kloster Steinheim in den Dominikanerorden aufgenommen.

Elisabeth von Blankenstein, nunmehr zum zweiten Mal Witwe, schenkte 1269 mit Genehmigung der Grafen Gottfried von Löwenstein und Hartmann von Grüningen, als den Oberherren über Steinheim, die Hälfte ihres Güterbesitzes dem Kloster Steinheim selbst. Auch bei diesem Rechtsakt wurde eine stattliche Zahl hochstehender Zeugen aufgeboten. Die zehn Siegler, die der darüber errichteten Urkunde ${ }^{71}$ Glaubwürdigkeit verliehen, wurden angeführt von Bischof Heinrich von Speyer und den Grafen Ulrich von Württemberg und Ulrich von Asperg. Zum Schluss siegelten Konrad von Heinriet und Engelhard von Weinsberg.

Man wird annehmen müssen, dass wir es hier mit Konrad II. von Heinriet zu tun haben, dessen Mitwirkung bei der Beurkundung der Schenkung ihn nicht daran hinderte, später von Priorin und Konvent einen Anteil an dem reichen Steinheimer Erbe, insbesondere an dem Freihof und dem Patronatsrecht zu fordern. Der darüber entstandene Streit wurde einem fünfköpfigen Schiedsgericht zur Entscheidung übergeben, das nach der Vorlage der Urkunde von 1235 gegen Konrad von Heinriet entschied und und das Erbe der Priorin und den Schwestern zusprach und Konrad deswegen Stillschweigen auferlegte ${ }^{72}$.

Konrad II. von Heinriet ist wohl nicht als Sohn Gerungs IV. und der Elisabeth anzusehen, da dies sonst in der Urkunde zum Ausdruck gekommen wäre. Somit ist er wohl ein Vetter der Burcsint von Heinriet, die zu dieser Zeit sicher schon dem Steinheimer Konvent angehörte. Ihr Grabstein ist immerhin das letzte Zeugnis einer Verbindung der Heinrieter zum Kloster Steinheim.

70 Dazu: Bernhard Theil, Steinheim vom 8. bis 18. Jahrhundert, in: Heimatbuch der Stadt Steinheim an der Murr, Steinheim 1980, S. 53-146, hier S.61-79.

71 WUB 7, Nr. 2064, S. 23-26.

72 WUB 7, Nr.2149, S. 91. 
Als Papst Alexander IV. mit Urkunde vom 20. Juni $1257^{73}$ das Zisterzienserinnenkloster Lichtenstern mit allen seinen Besitzungen in seinen Schutz nahm, werden unter diesen auch vier Hufen in Heinriet genannt. Somit hatten die Heinrieter auch eine Verbindung mit diesem, wohl 1242 gestifteten Kloster. Das Kloster Lichtenstern liegt bei Löwenstein auf einer Anhöhe über einem Bachlauf, der einen linken Zufluss der Sulm bildet. Von diesem Kloster Lichtenstern ${ }^{74}$ fehlen die üblichen Urkunden aus der Gründungszeit, für die das Jahr 1242 überliefert ist. Die einzigen Nachrichten über die Gründung bieten chronikalische Überlieferungen des 16. und 17. Jahrhunderts, die jedoch mit nicht geringen Schwierigkeiten behaftet $\operatorname{sind}^{75}$.

Nach der Überlieferung war Luitgard von Weinsberg die Stifterin von Lichtenstern. Sie war eine Tochter des Schenken Walter III. von Schüpf oder von Limpurg und Witwe Engelhards von Weinsberg. Der Ort der Klostergründung war, wie die Gründungsgeschichte mitteilt, in dem dückhen Wald und teils Erbgut der Kinder der Stifterin Luitgard von Weinsberg, andernteils Besitz eines Freiben Herren von Hohenrieth/ der was irer Kinde Mage, der Gab durch ir Bett/ und auch durch seiner Seel Hail sein Tail auch freilich und aigentlich an daß selb Closter ${ }^{76}$. Es handelt sich bei diesen Mitbesitzern der Örtlichkeit im Tuffingstal um die Herren von Heinriet, die als Verwandte der Weinsberger bezeichnet werden und somit Anteil an der Klostergründung nahmen, indem sie den Ort der Klostergründung, der ihnen zur Hälfte gehörte, zur Verfügung stellten. Bei den vier Hufen in Heinriet wird es sich wohl um eine zusätzliche Schenkung handeln.

Bei dem Zusammenwirken der Heinrieter mit der Tochter des Schenken Walter III. von Schüpf oder von Limpurg handelt es sich um zwei Familien, die König Heinrich (VII.), dem Sohn Kaiser Friedrichs II. nahe standen. Auf derselben Seite stand auch der Würzburger Bischof Hermann von Lobdeburg, der in seiner Diözese die Stiftung von Zisterzienserinnenklöstern, so auch von Lichtenstern, tatkräftig förderte. Freilich erfolgte die Gründung von Lichtenstern nach der Katastrophe Heinrichs (VII.), weshalb es für die Stifterfamilien nahe lag, Teile ihres Besitzes in klösterliche Hand zu geben, um so noch eine gewisse Verfügungsgewalt

73 WUB 5, Nr.1310, S.75-77, Nr.1310 mit Bd.11 S. 576.

74 Christa-Maria MACK, Die Geschichte des Klosters Lichtenstern von der Gründung bis zur Reformation (Göppinger Akademische Beiträge, Nr. 91), Göppingen 1975; Hermann EHMER, Zisterziensische Frauenklöster im baden-württembergischen Franken, in: Dieter R. Bauer (Hg.), Unter Beobachtung der heiligen Regel. Zisterziensische Spiritualität und Kultur im baden-württembergischen Franken (Forschungen aus Württembergisch Franken, Bd.48), Stuttgart 2002, S.49-58.

75 Die jüngste, aber zweifellos auf älteren Nachrichten gründende Fassung dieser Gründungsgeschichte bietet [Christoph Besold], Virginum Sacrarum Monimenta, Tübingen 1636, S. 423-426. Hierzu und zu den folgenden Ausführungen vgl. Mack (wie Anm. 74) S. $12-22$.

76 Besold (wie Anm. 75) S. 424. 
darüber zu behalten. In ähnlicher Weise diente die Gründung der Frauenzisterzen dem Bischof zum Ausbau seines geistlichen und weltlichen Zuständigkeitsbereichs, da er sich auf beiden Gebieten jeweils Rechte vorbehielt ${ }^{77}$.

Die Verbindung der Heinrieter mit dem Kloster Lichtenstern wird nicht zuletzt dadurch dokumentiert, dass mindestens zwei Männer aus dieser Familie im Kloster begraben wurden, deren Grabmäler offenbar noch im 16. Jahrhundert zu sehen waren $^{78}$. Möglich ist auch, dass eine Heinrieterin Nonne in diesem Kloster war, was bei der schlechten Überlieferungslage zumindest nicht ausgeschlossen werden kann.

\section{Die vergessene Stadt bei der Burg Heinriet}

In seiner Besprechung des Bands Württemberg von Keysers Städtebuch ${ }^{79}$ kriti- $^{-}$ siert Hans Jänichen unter anderem die Auswahl der behandelten Städte. Der profunde Kenner der südwestdeutschen Siedlungsgeschichte zählt acht Gemeinden auf, denen im Mittelalter Stadtrechte verliehen wurden oder doch einen Rechtszug städtischer Art hatten. Ferner nennt er 29 Gemeinden, deren städtischer Charakter feststeht und die in dem angezeigten Werk ebenfalls hätten behandelt werden müssen.

Selbstverständlich ist es eine Definitionsfrage, was in ein solches Sammelwerk aufgenommen werden müsste. Jänichen betont aber zu Recht, dass gerade die Misch- und Übergangsformen zwischen Dorf- und Stadtgemeinden für das Verständnis des südwestdeutschen Städtewesens von Bedeutung sind. Es wurden seitdem zahlreiche terminologische Erwägungen angestellt, um das Phänomen der erfolglosen Stadtgründungen in den Griff zu bekommen ${ }^{80}$. Die Diskussion soll hier nun nicht um einen neuen Begriff vermehrt werden, vielmehr wird hier ein bislang unbeachteter Stadtgründungsversuch vorgeführt. Die „Stadt“ bei der Burg Heinriet zählt nämlich nicht zu den Jänichenschen Desideraten. Sie ist freilich nicht einfach vergessen worden, vielmehr ist bislang niemand auf die Spuren, die sie hinterlassen hat, aufmerksam geworden.

77 Mack (wie Anm.74) S.19-21; Ehmer, Zisterziensische Frauenklöster (wie Anm. 74) S. 50.

78 Mack (wie Anm. 74) S. 15 nach Aufzeichnungen Gabelkovers.

79 Württembergisches Städtebuch, hg. von Erich KeYser (Deutsches Städtebuch, Bd.IV, 2), Stuttgart 1962. Besprechung von Hans JäNICHEN in: Zeitschrift für bayerische Landesgeschichte 26 (1963) S. 747-749.

80 Ausführlich dazu Nina KüHNLE, Wenn Städte sterben. Württembergische „Statuswüstungen“ des Mittelalters und der Neuzeit, in: ZWLG 73 (2014) S. 101 -136, hier S. $102-107$. 
Die Durchsicht des bereits erwähnten Lagerbuchs von $1524^{81}$ brachte einige auffällige Funde zutage. So wird die Statmur als Anstößer genannt, womit vermutlich die heute noch vorhandene Mauer gemeint ist. Es erscheinen hier ferner auch das Thor und der Statgraben. Dies sind Bezeichnungen, die bei einem Weiler doch ungewöhnlich sind und auf eine Vorgeschichte schließen lassen, die man sonst nicht vermutete. Die hier verwendeten Begriffe machen somit den Eindruck, als ob hier - auch baulich - einst eine Stadtgründung ins Werk gesetzt worden, aber nicht weiter gediehen ist. Diese Hinweise - auch wenn sie 200 Jahre jünger sind - bilden einen hinreichenden Verweis auf eine geplante Stadtgründung bei der Burg Heinriet am Vorhof. Der Platz war für eine solche Gründung günstig, befand er sich doch an der Straße von Schwäbisch Hall nach Heilbronn, an dem Weg, auf dem das Haller Salz zum Neckartal gelangte.

Der Vollständigkeit halber ist noch darauf hinzuweisen, dass die folgenden Lagerbücher des Amts Beilstein im Vorhof dieselben Stellennamen aufweisen, wie im Lagerbuch von 1524. Es ist das Lagerbuch von $1576 / 1577^{82}$ und das von $1687^{83}$. Dies ist freilich nicht weiter verwunderlich, weil es sich ja um Renovationen handelt, um Erneuerungen des jeweils vorhergehenden Lagerbuchs. Im Urkataster von 1834 sind diese Namen jedoch nicht mehr enthalten.

Die genannten Stellennamen sind aber nicht die einzigen Hinweise auf eine versuchte Stadtgründung. Eine Stadt braucht ein sakrales Zentrum intra muros, auch wenn die zuständige Pfarrkirche nur wenige Schritte extra muros steht. Dies zeigt etwa Großbottwar ${ }^{84}$ mit seiner alten Martinskirche vor den Mauern und der Allerheiligenkapelle in der Stadt, ebenso wie Vaihingen an der Enz ${ }^{85}$, mit der alten Peterskirche im Friedhof vor der Stadt und der Liebfrauenkapelle in der Stadt. Es darf deshalb der Bau und die Dotierung einer Kapelle bei der Burg Heinriet im Zusammenhang mit der Stadtgründung gesehen werden. Die Nachrichten über die Kapelle, von der erstmals 1323 die Rede ist, geben somit auch einen Hinweis für die zeitliche Ansetzung der Stadtgründung.

Am 17. August 1323 schenkte Friedrich d. Ä. von Heinriet mit Zustimmung seiner Erben zu seinem und seines verstorbenen Bruders Engelhard Seelenheil ein Viertel des großen und kleinen Zehnten in Pfarrei und Mark Heinriet dem Inhaber der Pfründe in der Kapelle seiner Burg Heinriet ${ }^{86}$. Als Mitsiegler erscheint hier

81 Altwürttembergische Lagerbücher (wie Anm.32) S.94. - KüHNLE (wie Anm. 80) S. 114, verweist daher zu Recht auf die Lagerbücher des 16. Jahrhunderts, in denen frühere Zustände konserviert sind.

82 HStAS H 1901/5 Bd. 2.

83 HStAS H 1901/5 Bd. 8.

${ }^{84}$ Gustav Hoffmann, Kirchenheilige in Württemberg (Darstellungen aus der Württembergischen Geschichte, Bd.23), Stuttgart 1932, S. 48 f.

${ }^{85}$ Ebd., S. 67.

${ }^{86}$ GLAK 67/889, Bl. 33r-v. 
Friedrich d.J., sein Sohn, den wir demnach Friedrich II. nennen. Konrad III. von Heinriet wollte hinter seinem Bruder nicht zurückstehen und schenkte 1325, ebenfalls mit Zustimmung seiner Erben, ein Viertel des großen und kleinen Zehnten in Pfarrei und Mark des Dorfes Heinriet für die Priesterpfründe der Kapelle in der Burg Heinriet. Als Konrads Erben siegeln mit seine Schwiegersöhne Eberhard von Staufeneck und Ulrich von Alfingen ${ }^{87}$. Erforderlich war jetzt noch die bischöfliche Genehmigung, die 1326 durch den zuständigen Pfarrer eingeholt wurde. Dies war Konrad von Helmbund, Kanoniker der Öhringer Kirche und Pfarrer der Kirche in Heinriet, der Bischof Wolfram von Würzburg mitteilte, dass der edle Herr Konrad von Heinriet mit seiner, des Pfarrers Zustimmung außerhalb seiner Burg Heinriet, aber innerhalb der Grenzen der Pfarrei, zur Vermehrung des Gottesdienstes eine Kapelle ohne Benachteiligung der Mutterkirche errichtet und diese reichlich mit Einkünften begabt habe. Die gemachten Angaben wurden von dem Nachbarpfarrer Hermann in Löwenstein bestätigt ${ }^{88}$.

Der bischöflichen Genehmigung stand nun nichts mehr im Wege, da die Pfarrkirche und deren Pfründe nicht beeinträchtigt, aber eine auskömmliche Pfründe für den an der Kapelle anzustellenden Kaplan gestiftet worden war. Allerdings waren die Zehntviertel in Pfarrei und Mark Heinriet, die Friedrich von Heinriet gestiftet hatte, Lehen vom Bischof von Würzburg, die Konrads hingegen nicht. Der Bischof bestätigte deshalb Schenkung Konrads ${ }^{89}$, Friedrich hingegen musste Ersatz schaffen. Dies geschah in der Weise, dass der Bischof zunächst Friedrich das bereits zur Pfründe gestiftete Zehntviertel übereignete und dieser dem Bischof zum Ausgleich $6 \mathrm{lb}$ h von verschiedenen Gütern, abzüglich eines Betrags von einigen Schillingen im Dörfchen Toneraswilre ${ }^{90}$ unterhalb der Burg Heinriet, sowie $2 \mathrm{lb}$ h Einkünfte aus Auenstein unter der Burg Helfenberg übergab ${ }^{91}$. Ein entsprechender Eintrag findet sich auch im Würzburger Lehenbuch ${ }^{92}$. Die Kapelle mit Frühmesspfründe bestand bereits 1330 und war mit einem Priester namens

87 GLAK 67/889, Bl. 19v-20 r.

88 GLAK 67/889, B1.20r-v. - Die unterschiedlichen Angaben, wonach hier von einer Kapelle außerhalb der Burg Heinriet die Rede ist, während in der Schenkungsurkunde Friedrichs vom 17. August 1323 und der Konrads vom 25. September 1325 von der Kapelle in der Burg gesprochen wird, haben nichts zu besagen. Es handelt sich um ein und dieselbe Kapelle.

${ }^{89}$ GLAK 67/889, Bl.33v-34 r.

90 Abgegangen.

91 GLAK 67/889 Bl.34 r-v. - Es wird damit auch die Lage der ausgegangenen Siedlung Toneraswilre im Buchbachtal unterhalb der Burg Heinriet angegeben.

92 Das älteste Lehenbuch des Hochstifts Würzburg. 1303-1345 (Quellen und Forschungen zur Geschichte des Bistums und Hochstifts Würzburg, Bd.25), bearb. von Hermann HoffMAnN, Würzburg 1972-1973, Nr. 2509. - Hier werden die genannten Beträge genauer angegeben. Es handelt sich um $6 \mathrm{lb}$ b minus $5 \beta$ in villula Toneraswiler sub castro Hehenrit et in villula Ostheim sub Castro Helfemberg redditus $2 \frac{1}{2} \mathrm{lb} h$. 
Götz besetzt ${ }^{93}$. Diese Katharinenkapelle erscheint im Lagerbuch von $1524^{94}$, denn der dort genannte Kirchboff, der einer Kapelle eigentlich nicht zusteht, da sich die Pfarrkirche ja in Unterheinriet befindet, ist auf diese zu beziehen. Die Würzburger Diözesanmatrikel aus der Mitte des 15. Jahrhundert führt deshalb die Katharinenkapelle im Vorhof nicht auf, hingegen die Pfarrkirche in Hebenriet (Unterheinriet $)^{95}$.

Wir müssen daher Friedrich I. und Konrad III. von Heinriet als Stadtgründer ansehen, wenngleich ihre - versuchte - Gründung, die wohl ins zweite Jahrzehnt des 14. Jahrhunderts anzusetzen ist, erfolglos blieb. Aber sie müssen die Stadtgründer sein, denn die Grafen von Löwenstein, die alsbald in den Besitz von Burg und Herrschaft Heinriet gelangten, können kein Interesse daran gehabt haben, am Vorhof eine Stadt entstehen zu lassen, die ihrer eigenen Stadt Löwenstein Konkurrenz gemacht hätte. So bleiben eben nur die beiden Heinrieter Brüder als - wenn auch erfolglose - Stadtgründer, deren Pläne sich noch in den im Lagerbuch von 1524 genannten Stellennamen widerspiegelt.

\section{Der Ausverkauf der Herrschaft Heinriet}

Die Stiftung der Katharinenkapelle bei der Burg Heinriet war somit ein Zeichen weitergehender Planungen in Richtung auf eine Stadtgründung. Dies müsste eigentlich als Zeichen wirtschaftlichen Wohlstands angesehen werden. Doch dem war nicht so, denn Konrad von Heinriet und sein Schwiegersohn Eberhard von Staufeneck verkauften am 6. Januar 1330 wegen ihrer Schulden die Hälfte der Herrschaft Heinriet um $600 \mathrm{lb}$ h an den Grafen Nikolaus von Löwenstein. Diese Hälfte bestand aus dem Halbteil der Burg und des Vorhofs zu Heinriet, den Rechten an der Kapelle und der Frühmesspfründe im Vorhof, das Dorf (Unter-)Heinriet mit Kirchsatz und alle Zugehörungen von Burg, Vorhof und Dorf, sei es im Heinrieter Tal gelegen oder anderswo. Dazu gehörten ferner zwei Höfe zu Ilsfeld, zwei zu Wüstenhausen (Husen), ein Hof zu Willsbach (Wyresbach), Berwinkel und die anderen Weiler auf dem Wald gegen Murrhardt gelegen, sie seien Lehen vom Kloster Murrhardt oder nicht, mit Zubehör. Hinzu kamen noch die die obere und die niedere Fischenz in der Jagst zu Untergriesheim, die Fischenz zu Offenau (Uffenheim), die man nennt zu dem wage im Neckar zwischen Offenau und Wimpfen. Die Güter sollen dem Grafen Nikolaus zu Eigen gehören, ausgenommen die Weiler auf dem Walde gegen Murrhardt. Diese sollen ihm als Lehen - offenbar

93 GLAK 67/890, B1.35v.

94 Altwürttembergische Lagerbücher (wie Anm. 32) S. 94. Demnach hatte die Herrschaft diese Pfründe zu verleihen, ebenso wie die Pfarrpfründe in Unterheinriet.

95 Franz J. Bendel, Die Würzburger Diözesanmatrikel aus der Mitte des 15. Jahrhunderts, in: Würzburger Diözesangeschichtsblätter 2 (1934) S. I-XXX, 1-46, hier S. 12. 
vom Kloster Murrhardt - gehören ${ }^{96}$. Der Abt von Murrhardt siegelte daher mit; ebenso der Ritter Eberhard von Staufeneck, der Schwiegersohn des Konrad von Heinriet. Dessen Frau Elsbeth und beider Sohn Georg willigten eigens noch in den Verkauf der halben Burg und des Vorhof Heinriet an den Grafen Nikolaus von Löwenstein ${ }^{97}$.

Der Verkauf wurde, wohl auf Betreiben des Käufers, des Grafen Nikolaus von Löwenstein, rechtlich abgesichert, denn neben der eben erwähnten Urkunde wurde noch eine weitere darüber ausgestellt, und zwar vom Bischof von Würzburg. Gewiß war er der Lehenherr einiger Güter und Einkünfte, die Konrad von Heinriet dem Grafen Nikolaus von Löwenstein verkauft hatte, doch ging es in dieser Urkunde nicht nur um dessen Zustimmung, vielmehr beurkundete der Bischof am 16. Januar $1330^{98}$ den gesamten Verkauf. Damit lernen wir die Verkaufsobjekte näher kennen. Genannt wird hier neben der Hälfte der Burg Heinriet mit dem Vorhof (suburbium) und des Dorfes Heinriet auch Oberheinriet, Schwengelhausen (Swengelbusen), Besenhausen (Besenhusen), und Gerhausen (Gerbusen) sowie zwei Allode in Ilsfeld, zwei Allode in Wüstenhausen (Husen), ein Allod in Happenbach, eines in Willsbach (Wilrspach) sowie jährliche Einkünfte von 13 Pfd. h. in Berwinkel und den anderen dazu gehörigen Weilern, außerdem drei Seen bei der Burg Heinriet und drei Fischrechte mit allen Zugehörungen.

Bei den drei Siedlungen Schwengelhausen, Besenhausen und Gerhausen handelt es sich um seither ausgegangene Orte, die heute noch durch entsprechende Flurnamen westlich von Ober- und Unterheinriet, jenseits des Schozachlaufs lokalisiert werden können ${ }^{99}$. Bei den genannten Alloden oder Eigengütern in Ilsfeld, Wüstenhausen, Happenbach und Willsbach handelt es sich um die in der Verkaufsurkunde daselbst genannten Höfe. Neu sind die drei Seen unterhalb der Burg Heinriet, also im oberen Buchbachtal, wo die durchstochenen Staudämme teilweise noch heute zu sehen $\operatorname{sind}^{100}$. Die drei Seen waren offenbar der Ersatz für die jetzt nicht mehr genannten Fischgründe in der Jagst und im Neckar.

Während die Mitwirkung des Bischofs von Würzburg als Lehensherrn durchaus einsichtig ist, überrascht doch die Tatsache, dass auch Kaiser Ludwig der Bayer mit

96 GLAK 67/890, Bl.35v, dazu der Fertigungsbrief vom 13. Januar 1330; GLAK 67/890, Bl. 19r. - Zur Sache vgl. Gerhard FriTz, Geschichte der Grafschaft Löwenstein und der Grafen von Löwenstein-Habsburg vom späten 13. bis zur Mitte des 15. Jahrhunderts (Forschungen aus Württembergisch Franken, Bd.29), Sigmaringen 1986, S. 48.

97 GLAK 67/890, Bl. 19v.

98 Regest: GLAK 67/889, Bl.35r-v. Druck: Kremer, Abhandlung (wie Anm.9) S. $358-360$.

${ }_{99}$ Eberhard Schweizer, Wüstungen in Heinriet, in: Untergruppenbach. Heimatbuch der Gemeinde Untergruppenbach, Stuttgart 1992, S.137f.

100 Werner Heim, Zur Geschichte des Bleichsees, in: Schwaben und Franken. Heimatgeschichtliche Beilage der Heilbronner Stimme 24 (1977) Nr. 8. 
einer am 5. August 1330 in Hagenau ausgestellten Urkunde ${ }^{101}$ den Verkauf bestätigte. Offensichtlich war den Beteiligten bewusst, dass die Herrschaft Heinriet ursprünglich Königsgut war, das die Herren von Heinriet vor alters bekommen hatten, wenngleich es wohl schon lange nicht mehr zu den üblichen Belehnungen gekommen war. Es musste also im Interesse des Käufers, des Grafen Nikolaus von Löwenstein sein, sich den Kauf auch vom Kaiser bestätigen zu lassen ${ }^{102}$.

Einem Ausverkauf widerspricht auf den ersten Blick, dass Konrad von Heinriet 1327 Burg und Stadt Neudenau (Nidenawe) mit Zubehör von Konrad von Weinsberg auf Wiederkauf erworben hat ${ }^{103}$. Man könnte somit annehmen, dass es sich bei dem Verkauf der ihm gehörigen Hälfte der Herrschaft Heinriet um eine Besitzverlagerung in das an der Jagst, ein Stück Wegs oberhalb ihrer Mündung in den Neckar gelegene Neudenau handelte. Doch verhielt sich die Sache anders; Neudenau war zu jener Zeit ein Objekt unter anderen in einer komplizierten finanziellen Transaktion zwischen Konrad von Weinsberg und Ulrich von Hanau, bei der es ursprünglich um die von dem Hanauer seinem Schwager Konrad von Weinsberg versetzte Burg Dorfelden (jetzt Niederdorfelden bei Hanau) ging. Konrad von Heinriet spielte in dieser Geschichte lediglich die Rolle eines Mittelsmannes und ging vermutlich leer aus. Neudenau kam nämlich 1330 in die Hand von Burkhard Sturmfeder und gelangte 1364 an das Erzstift Mainz ${ }^{104}$.

Der Verkauf der halben Herrschaft Heinriet hatte noch ein Nachspiel, das sich ausgerechnet auf die Burgkapelle mit ihrer Frühmeßpfründe bezog. Es kam zu einem Rechtsstreit zwischen dem Grafen Nikolaus von Löwenstein und Friedrich d. Ä. von Heinriet über die Frage, wem nun das Präsentationsrecht der Pfründe der Kapelle im Vorhof Heinriet (in suburbio Hebenriet) zustehe. Die Entscheidung des Falls wurde dem Dekan Heinrich des Kapitels Weinsberg zusammen mit dem Schatzmeister Diether der Kirche in Wimpfen und Dekan Heinrich in Großbottwar (Botbor) übertragen. Diese gaben, nachdem sie den Fall in Heilbronn beraten hatten, ihre Entscheidung am 25. Mai 1333 bekannt ${ }^{105}$. Sie sprachen das Präsentationsrecht dem Grafen Nikolaus zu, denn in dem von Konrad von Heinriet, dem

101 GLAK 67/890, Bl.36r-v; danach: Regesta Imperii VII, 113, wo allerdings die Identifizierung der Burg mit „[Unter-]Heinriet“ zu berichtigen ist.

102 Die Notiz, wonach Kurfürst Rudolf II. von der Pfalz 1331 die Burg Hohenriedt (ob Heinriet?) mit dem Patronatsrecht erworben habe (Regesten der Pfalzgrafen am Rhein 1214-1508, Bd.1: 1214-1400, bearb. von Adolf KocH/Jakob Wille, Innsbruck 1894, Nr.2129) beruht auf: Christoph von Chlingensperg, Processus Historico-Juridicus in Causa Successionis Palatinae, Ingolstadt 1771, S.91, und dürfte auf einen Irrtum zurückgehen. Wäre diese Notiz richtig, würde sie voraussetzen, dass Graf Nikolaus von Löwenstein seine Erwerbung umgehend an die Pfalz weiterverkauft hätte, was jedoch, wie der folgende Streit um die Kapelle zeigt, offensichtlich nicht der Fall war.

${ }_{103}$ Nachweis im Archiv Amorbach nach Albert KRIEGER, Topographisches Wörterbuch des Großherzogtums Baden, Bd.2, Karlsruhe 1905, Sp. 277.

104 Vgl. Der Landkreis Heilbronn (wie Anm. 26) S. $233 \mathrm{f}$.

105 GLAK 67/889, Bl.21r-22r. 
Bruder Friedrichs, getätigten Verkauf seiner Hälfte der Burg Heinriet, des Vorhofs und anderem, sei auch das Patronatsrecht der Pfarrkirche in Heinriet, also das Recht, den Pfarrer und den Priester an der Altarpfründe im Vorhof der Burg Heinriet zu präsentieren, inbegriffen. Hierfür hatte der Verkäufer den Bischof Wolfram von Würzburg um Bestätigung gebeten und diese auch erlangt. Der von Konrad von Heinriet in der Kapelle eingesetzte Pfaff Götz von Mosbach sei von Bischof Wolfram bestätigt und werde auch von dem Grafen anerkannt.

Die andere Hälfte der Burg Heinriet mit Zubehör verkauften Rudolf von Heinriet, seine Frau Brigitta, und ihr Sohn Rudolf am 18. März 1364 wegen Verschuldung an Graf Albrecht von Löwenstein um $1400 \mathrm{lb} \mathrm{h}^{106}$. Es handelte sich um die Hälfte der Burg Heinriet, die Kapelle, die Pfründe in der Burg und den Vorhof halb, die Güter und Mühle zu Dofranswiler ${ }^{107}$, die Güter zu Buch, zu der Ebene, zu dem Hetzelberg, zu Eptzenwiler ${ }^{108}$, die Mühle am Knollensee, den See zur Hanwiese und was sie zur Hanwiese auf und ab hatten, den Hof im Dorf Heinriet mit aller Zugehörde, die Bäche auf und ab bis vor das Dorf in die Furt, die Weingärten am Sandberg, an der Spatzenhalde und am Schnarrenberge, das Gericht, die Vogtei, die Eigenleute und alle weiteren Rechte.

Auch dieses Mal wurde nicht versäumt, die kaiserliche Bestätigung zu dem getätigten Verkauf einzuholen. Rudolf von Heinriet, seine Frau Brigitta und Rudolf, ihr Sohn, baten noch am selben Tag Kaiser Karl IV. als Lehensherrn, seine Zustimmung zu dem Verkauf zu geben ${ }^{109}$. Das Bewusstsein, dass es sich bei der Herrschaft Heinriet um ein Reichslehen handelte, war also immer noch lebendig.

Nach diesen Verkäufen scheinen die Heinrieter ihren Besitzschwerpunkt nach Helfenberg verlegt zu haben, das Konrad von Heinriet am 19. April 1456 an den Grafen Ulrich IV. von Württemberg verkaufte. Der Verkauf umfasste das Schloss Helfenberg und den Burgstall hinter dem jetzigen Schloss, das man Althelfenberg nennt, mit dem Berg, mit Leuten, Gülten und Gütern. Die Kaufsumme betrug $1200 \mathrm{fl}$. Das Ganze sieht wieder nach einem Ausverkauf aus, denn der Heinrieter verkaufte offensichtlich alles, was zum Helfenberg gehörte, wie die eingehende Beschreibung der einzelnen Verkaufsgegenstände in der Urkunde zeigt.

106 GLAK 67/890, Bl.36r.

107 Sicher identisch mit dem oben genannten Toneraswilre. Die eine oder die andere Form dürfte auf eine Verschreibung zurückgehen.

108 Abgegangen, nicht weiter zu identifizieren.

109 GLAK 67/890, Bl.36r-v. 


\section{Das Ende der Herren von Heinriet}

Die Heinrieter waren längst im Stand der Niederadligen angelangt. Aus den Edelfreien der Stauferzeit waren Fehdereiter im Dienste der Territorialherren geworden. Vier Jahre nach dem Verkauf von Helfenberg, am 30. April 1460, fiel Konrad von Heinriet im Verlauf der Pfälzer Fehde zwischen Württemberg und der Kurpfalz zusammen mit Kaspar Spät in einem Gefecht zwischen Wüstenhausen und Helfenberg, wie die den beiden Gefallenen gewidmete Inschrift des Gedächtnisbildes in der Alexanderkirche in Marbach dartut ${ }^{110}$ (Abb.4). Philipp von Heinriet, dessen Verwandtschaft mit Konrad unklar ist, fiel am 8. August 1462 in Diensten des Bischofs Johann von Würzburg in dessen Fehde gegen Markgraf Albrecht von Brandenburg vor Marktbreit. Er wurde - als der letzte Heinrieter - in der Karmeliterkirche in Heilbronn begraben ${ }^{111}$.

110 Die Inschriften des Landkreises Ludwigsburg (wie Anm.68) Nr.89, S.60f. mit Abb. 47, 48. Zu dem Gefecht vgl. Thomas Fritz, Ulrich der Vielgeliebte (1441-1480). Ein Württemberger im Herbst des Mittelalters. Zur Geschichte der württembergischen Politik im Spannungsfeld zwischen Hausmacht, Region und Reich (Schriften zur südwestdeutschen Landeskunde 25), Leinfelden-Echterdingen 1999, S.219. Vgl. auch ebd. S. 167 zum Verkauf der Burg Helfenberg an Württemberg 1456. - Fritz gebraucht die Namensform Hohenriet. Bei ausgestorbenen Familien ist es gerade bei der Vielzahl der für die Heinrieter überlieferten Namensformen ratsam, sich an die gegenwärtige Schreibung des Ortes zu halten, nach dem sie sich genannt haben.

111 Lorenz FrIEs, Chronik der Bischöfe von Würzburg 742-1495, Bd. 4, hg. von Ulrich WAGNER/Walter ZIEGLER, Würzburg 2002, S. 198. 


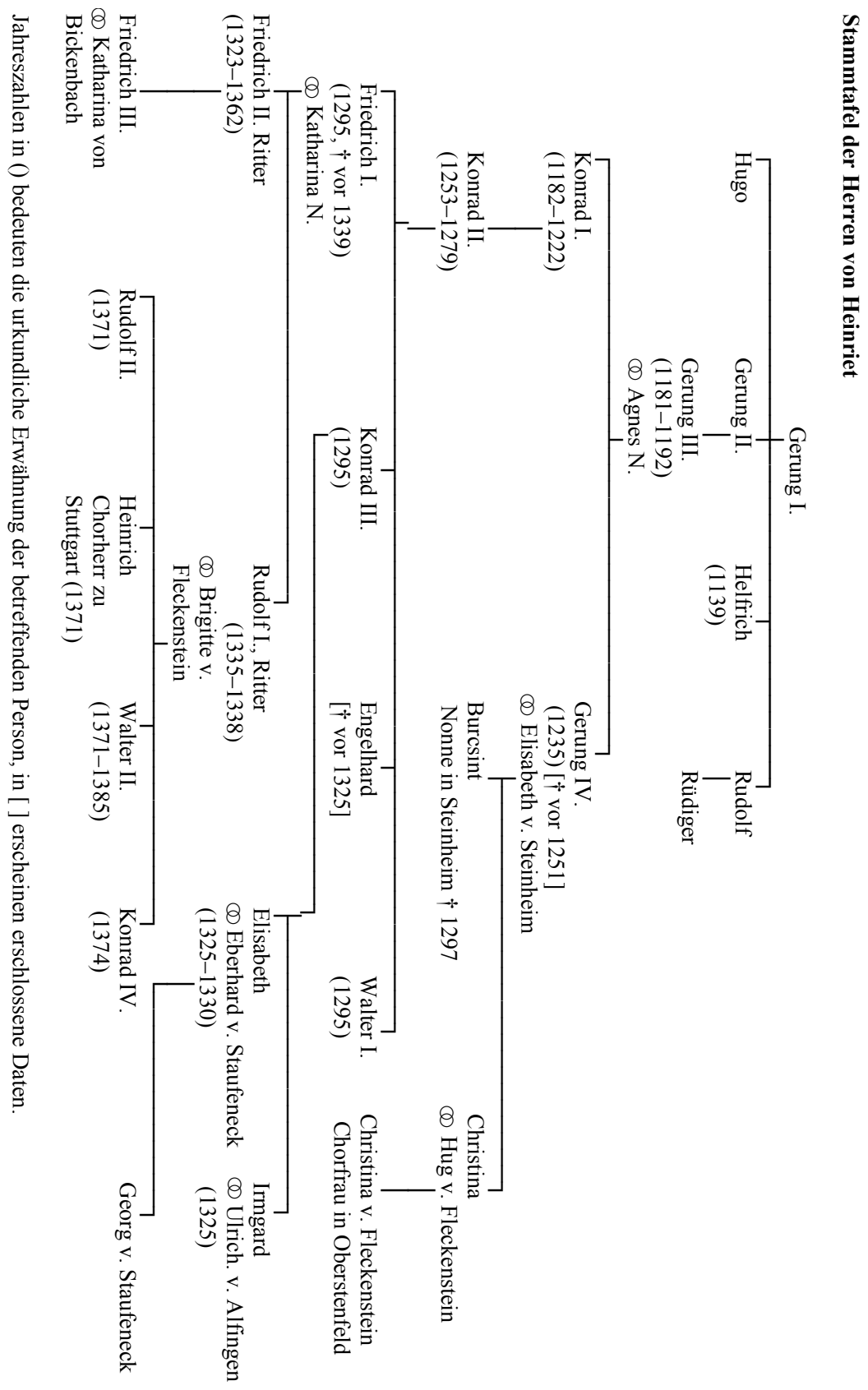

